\title{
Clinical Consequences of Withholding Versus Administering Renin-Angiotensin-Aldosterone System Antagonists in the Preoperative Period
}

\author{
David J. Rosenman, MD, Msc ${ }^{1,3,4}$ \\ Furman S. McDonald, MD, $\mathrm{MPH}^{3,4}$ \\ Jon 0. Ebbert, MD, MSc ${ }^{3,4}$ \\ Patricia J. Erwin, MLS ${ }^{2,4}$ \\ Matthew LaBella ${ }^{5}$ \\ Victor M. Montori, MD, MSc ${ }^{1,3,4}$ \\ ${ }^{1}$ Knowledge and Encounter Research Unit, Mayo \\ Clinic College of Medicine, Rochester, Minnesota \\ ${ }^{2}$ Mayo Library, Mayo Clinic College of Medicine, \\ Rochester, Minnesota \\ ${ }^{3}$ Department of Medicine, Mayo Clinic College of \\ Medicine, Rochester, Minnesota \\ ${ }^{4}$ Mayo Clinic College of Medicine, Rochester, \\ Minnesota \\ ${ }^{5}$ State University of New York at Geneseo, \\ Geneseo, New York
}

BACKGROUND: Hospitalists involved in perioperative care either stop or continue until the day of surgery renin-angiotensin-aldosterone system antagonists (either angiotensin-converting enzyme inhibitors [ACEI] or angiotensin II receptor subtype 1 antagonists [ARA]) in patients who use these agents chronically. This practice variation reflects uncertainty regarding the risks and benefits of either approach.

PURPOSE: The purpose of this study was to assess the clinical consequences of preoperatively continuing versus withholding ACEI/ARAs in patients treated chronically with these agents.

DATA SOURCES AND STUDY SELECTION: We comprehensively searched 7 major electronic databases, considered references from selected reviews, hand-searched journals, and communicated with experts. We included randomized trials and observational studies.

DATA EXTRACTION: We evaluated the relative risk (RR) of hypotension requiring vasopressors and of myocardial infarction in patients who did or did not receive an immediate preoperative dose of ACEI or ARA.

DATA SYNTHESIS: Random-effects meta-analysis from 5 studies totaling 434 patients suggested that patients receiving an immediate preoperative ACEI/ARA dose were more likely (RR 1.50, 95\% CI 1.15-1.96) to develop hypotension requiring vasopressors at or shortly after induction of anesthesia. Sufficient data were not available to assess other outcomes.

CONCLUSIONS: Preoperative administration of ACEI/ARAs increases intraoperative hypotension. The long-term clinical consequences of continuing versus withholding preoperative ACEI/ARAs are unknown. This uncertainty stems in part from the absence to date of randomized trials designed specifically to examine patient-important consequences of this decision. Journal of Hospital Medicine 2008;3:319-325. ㅇ 2008 Society of Hospital Medicine.

KEYWORDS: ACE-I, ARA, ARB, anesthesia, angiotensin converting enzyme inhibitor, angiotensin receptor blocker, outcomes, perioperative, preoperative.

C linicians commonly use renin-angiotensin-aldosterone-system (RAAS) antagonists such as angiotensin-converting enzyme inhibitors (ACEIs) and angiotensin II receptor subtype 1 antagonists (ARAs) to treat hypertension, congestive heart failure, and diabetic nephropathy. Hospitalists and other clinicians involved in the preoperative care of patients treated chronically with these agents are faced with the uncertainty of whether to continue these medications immediately prior to surgery.

The concern among those who recommend holding therapy is that pharmacologic suppression of the RAAS in patients undergoing general anesthesia may lead to severe or refractory 
(to intravenous fluid support) hypotension requiring vasopressors. On the other hand, if complications are no more likely when continuing one of these agents up to the day of surgery, withholding it could represent an unnecessary and potentially harmful intervention (eg, when a clinician caring postoperatively for a patient forgets to restart it). Although several studies have attempted to address this dilemma, a systematic and comprehensive summary of the pertinent evidence has not been published.

In this systematic review and meta-analysis, we sought to summarize the best available evidence about the relative incidence of patient-important outcomes ${ }^{1}$ in patients who do or do not receive ACEI/ARA therapy on the day of their nonemergent surgery.

\section{METHODS}

We report this protocol-driven review in accordance with the Quality of Reporting of Meta-analyses (QUOROM) standards for reporting systematic reviews of randomized trials. ${ }^{2}$

\section{Search Strategy}

In collaboration with an expert reference librarian (P.J.E.), we designed a search strategy that included the electronic databases MEDLINE, EMBASE, CINAHL, Web of Science, Current Contents, CENTRAL, DARE, and SCOPUS from 1981 (when captopril, the first ACEI, was approved by the FDA) until March 2006. We also reviewed the reference lists of included articles, retrieved articles from our personal files, and consulted with anesthesiologists and hospitalists with an interest in perioperative care in order to identify unpublished studies or studies missed by our strategy.

\section{Study Selection}

Eligible studies were prospective cohort studies or randomized controlled trials enrolling adult patients (ie, most patients $>18$ years) undergoing nonemergent surgery and using ACEI or ARA chronically and assessing the effect of withdrawing or continuing these agents up to the morning of surgery. Eligible studies measured and reported either events of great patient importance (death, myocardial infarction, transient ischemic attack or stroke, and hepatic or renal failure) or of potentially less importance such as unplanned admission to the intensive care unit or treatment-requiring hypotension, arrhythmias, or hyperkalemia.

\section{Study Selection}

Two reviewers (D.J.R. and F.S.M.) independently screened the titles and abstracts for potential inclusion and retrieved potentially eligible articles for full-text evaluation. Two reviewers (D.J.R. and M.L.B.) working in duplicate independently selected studies for inclusion. The reviewers were in agreement for full text inclusion $100 \%$ of the time.

\section{Data Extraction}

Two hospitalists with experience in perioperative care and trained in clinical research (D.J.R. and F.S.M.) working independently and in duplicate extracted data from each eligible article using a standardized structured data extraction form. We extracted information about the study authors and publication, the patients (numbers in each group, indications for chronic ACEI/ARA therapy, type of surgery, agents used for anesthesia), event rates of surgical and perioperative complications (death, stroke, myocardial infarction, unplanned admission to the intensive care unit, treatment-requiring hypotension, arrhythmias, or hyperkalemia), and relevant periods (e.g., between last dose of ACEI/ ARA and surgery, between surgery and clinical end points, total follow-up). When key information was not available in the published report, we contacted authors by electronic mail. We made 2 attempts to contact authors who failed to respond. Three of the 4 authors contacted responded with the requested information.

\section{Quality Assessment}

For randomized trials, we noted whether authors reported adequate allocation concealment, blinding of patients, clinicians, data collectors, data analysts, outcome assessors, and loss to follow-up. The same reviewers (D.J.R. and F.S.M.) assessed study quality and were in agreement for each article and each domain of quality (kappa statistic in each case was 1.0). For cohort studies we noted details of cohort selection and comparability according to the Newcastle-Ottawa approach. ${ }^{3}$

\section{Statistical Analysis}

We used a DerSimonian and Laird random effects $\operatorname{method}^{4}$ to conduct meta-analyses across eligible 
outcomes. Random effects meta-analysis incorporates both within-study and between-study variability. We chose a random effects approach because of the important degree of clinical heterogeneity expected between the included studies. For rare events we followed the approach by Sweeting et al. for the choice of a continuity correction factor. ${ }^{5}$ We report the pooled relative risk and the associated $95 \%$ confidence interval.

\section{Inconsistency and Subgroup Analyses}

To ascertain the magnitude of inconsistency across trials, we measured the $\mathrm{I}^{2}$ statistic, an estimate of the proportion of the overall betweenstudy variability that is not a result of random error or chance but of true clinical heterogeneity. ${ }^{6}$ When possible, we explored subgroup analyses to explain heterogeneity, including subgroups defined by type of surgery (cardiovascular versus noncardiovascular), timing of measurement of outcomes (in relation to anesthesia induction postoperatively), and type of agent (ACEI or ARA). We estimated the difference in treatment effects between subgroups by testing the hypothesis of treatment-subgroup interaction with a nominal significance level of $5 \%{ }^{7}$

\section{RESULTS}

\section{Search Results}

The 509 titles reviewed included 410 titles produced by electronic searches and an additional 99 titles from other sources (Fig. 1).

\section{Study Characteristics}

Table 1 summarizes the characteristics of the 5 included studies ( $\mathrm{n}=434$ patients). Myocardial infarction was an end point in 3 studies (Brabant, Bertrand, and Comfere); 1 event was reported in the "withheld" arm of each of these studies (none in the "continuing" arms). Hypotension requiring vasopressors was reported in all 5 studies. The other end points of interest were reported sparsely. There was considerable heterogeneity across studies regarding follow-up period, which ranged from ending at incision to ending at dismissal from the hospital.

\section{Methodological Quality of Included Studies}

Table 2 describes the methodological quality, as reported, of the included studies. Allocation concealment was unclear in 2 of the 3 randomized

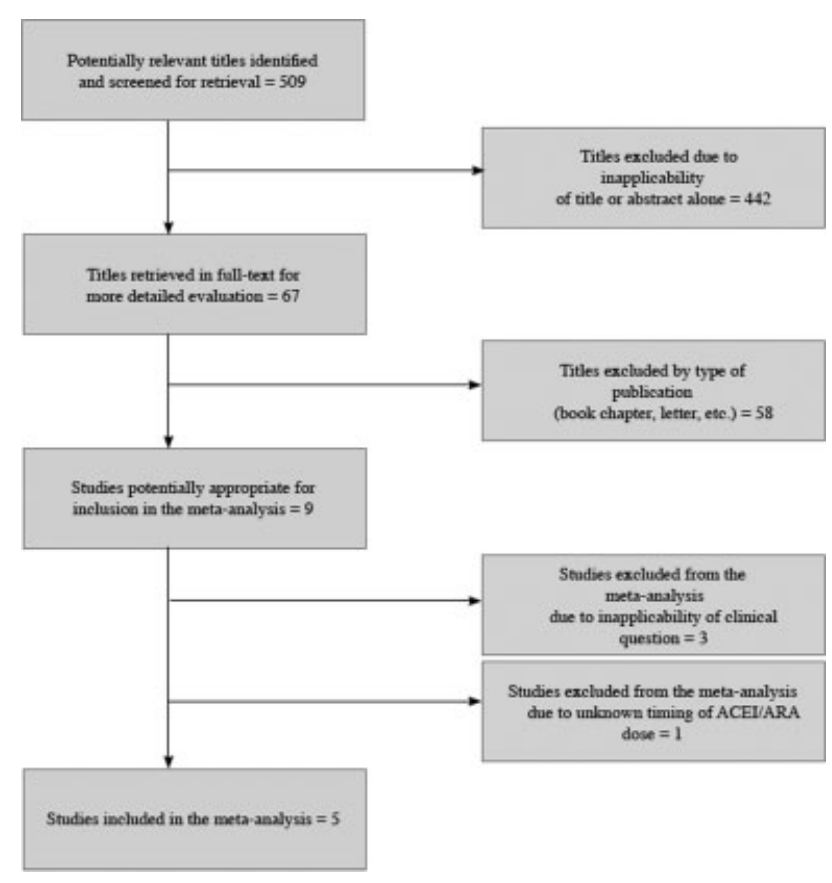

FIGURE 1. Flow diagram of study identification and selection.

trials. Details of blinding either were not reported or otherwise were unclear in 2 of these 3 studies. Only 1 study specified the extent of loss to followup. ${ }^{8}$ In 1 of the observational studies, ${ }^{9}$ details of cohort selection were generally appropriate. The 12 patients examined in another study ${ }^{10}$ had been scheduled consecutively for surgery. Both studies controlled for a variety of demographic and other key variables. Duration of follow-up ranged from 3 days after surgery (for ECG) ${ }^{10}$ to as long as duration of hospitalization. ${ }^{9}$

\section{Meta-analyses}

Pooled results suggested that patients receiving the immediate preoperative ACEI/ARA dose were more likely (RR $1.51,95 \%$ CI 1.14-2.01) to develop hypotension requiring vasopressors at or shortly after induction of anesthesia (Fig. 2A). There was important inconsistency between studies $\left(\mathrm{I}^{2}=\right.$ $59 \%)$. The pooled effect derived from randomized trials $(\mathrm{RR}=2.26,95 \%$ CI 0.84-6.12) seemed greater than that derived from the 2 observational studies $(\mathrm{RR}=1.33,95 \%$ CI 1.02-1.73), but the treatment-study design interaction was not significant $(P=.3)$. Similarly, other subgroup explorations were not contributory. 
TABLE 1

Characteristics of included studies

\begin{tabular}{|c|c|c|c|c|}
\hline Author/Year & Patients (n) & Indication for ACEI/ARA & Type of surgery & End points measured \\
\hline \multicolumn{5}{|l|}{ Randomized trials } \\
\hline Bertrand, $2001^{11}$ & 19 continued 18 withheld & Hypertension & Elective major vascular & $\begin{array}{l}\text { Hypotension, need for vasoactive } \\
\text { drugs (at or shortly after induction) }\end{array}$ \\
\hline Coriat, $1994^{8}$ & 21 continued 30 withheld & Hypertension & Peripheral vascular (>2 hours) & $\begin{array}{l}\text { Systolic blood pressure (at or shortly } \\
\text { after induction), plasma ACEI and } \\
\text { catecholamine levels }\end{array}$ \\
\hline Pigott, $1999^{17}$ & 20 continued 20 withheld & $\begin{array}{l}\text { Hypertension }(\mathrm{n}=17) \text {; previous } \\
\text { myocardial infarction }(\mathrm{n}=23)\end{array}$ & Coronary artery bypass graft & $\begin{array}{l}\text { Arterial pressure (at or shortly after } \\
\text { induction), cardiac index, systemic } \\
\text { vascular resistance, use of } \\
\text { vasoactive drugs }\end{array}$ \\
\hline \multicolumn{5}{|c|}{ Observational studies } \\
\hline Brabant, $1999^{10}$ & 12 continued 27 withheld & $\begin{array}{l}\text { Previous myocardial infarction }(\mathrm{n}= \\
\text { 6); diabetes mellitus }(\mathrm{n}=6 ; \mathrm{n} \text { with } \\
\text { diabetic nephropathy unknown); } \\
\text { hypertension ( } \mathrm{n}=\text { unknown) }\end{array}$ & Elective vascular surgery & $\begin{array}{l}\text { Blood pressure (at or shortly after } \\
\text { induction) }\end{array}$ \\
\hline Comfere, $2005^{9}$ & 144 continued 123 withheld & Hypertension & Noncardiovascular & $\begin{array}{l}\text { Blood pressure (at or shortly after } \\
\text { induction), unplanned ICU } \\
\text { admissions, hemodynamic } \\
\text { instability in the PACU (ABP or HR } \\
\text { out of range), acute renal } \\
\text { impairment, TIA, stroke, myocardial } \\
\text { ischemia/infarction, and death }\end{array}$ \\
\hline
\end{tabular}

ACEI/ARA, renin-angiotensin-aldosterone-system antagonists (either angiotensin-converting enzyme inhibitors [ACEIs] or angiotensin II receptor subtype 1 antagonists [ARAs]); ICU, intensive care unit; PACU, postanesthesia care unit; $\mathrm{ABP}$, arterial blood pressure; $\mathrm{HR}$, heart rate; TIA, transient ischemic attack.

TABLE 2

Quality of Included Studies

Randomized trials

\begin{tabular}{lll}
\hline & Allocation concealment & Blinding \\
\hline Bertrand, $2001^{11}$ & Unclear & Unclear \\
Coriat, $1994^{8}$ & Unclear & None \\
Pigott, $1999^{17}$ & Adequate & Investigator, cardiac anesthetists, perfusionists, and recovery staff were \\
& blinded to allocation. Blinding not reported for other data collectors, \\
& assessors of outcome, or data analysts \\
& & Not reported \\
\hline
\end{tabular}

Observational studies

\begin{tabular}{|c|c|c|}
\hline & Details of cohort selection & Comparability of cohorts \\
\hline Brabant, $1999^{10}$ & $\begin{array}{l}\text { Appropriate } \\
\text { Cohort somewhat representative of the adult population } \\
\text { undergoing nonemergent surgery. The unexposed cohort was } \\
\text { drawn from the same community as the exposed cohort }\end{array}$ & $\begin{array}{l}\text { Similar with } 2 \text { exceptions: compared with the ACEI-withheld } \\
\text { group, the ARA-given group contained more than twice the } \\
\text { proportion of patients with previous myocardial } \\
\text { revascularization } \\
\text { Compared with the ARA-given group, the ACEI-withheld } \\
\text { group contained more than twice the proportion of patients } \\
\text { with diabetes mellitus }\end{array}$ \\
\hline Comfere, $2005^{9}$ & $\begin{array}{l}\text { Appropriate } \\
\text { Cohort somewhat representative of the adult population } \\
\text { undergoing nonemergent surgery (referral center population may } \\
\text { not truly represent overall population). The unexposed cohort was } \\
\text { drawn from the same community as the exposed cohort. Data } \\
\text { were extracted from a secure record }\end{array}$ & $\begin{array}{l}\text { Adequate } \\
\text { This study controls for a variety of demographic and other } \\
\text { variables }\end{array}$ \\
\hline
\end{tabular}


A)

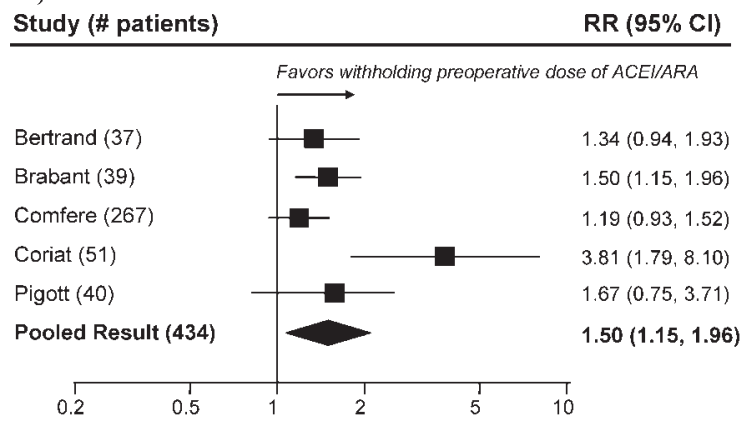

B)

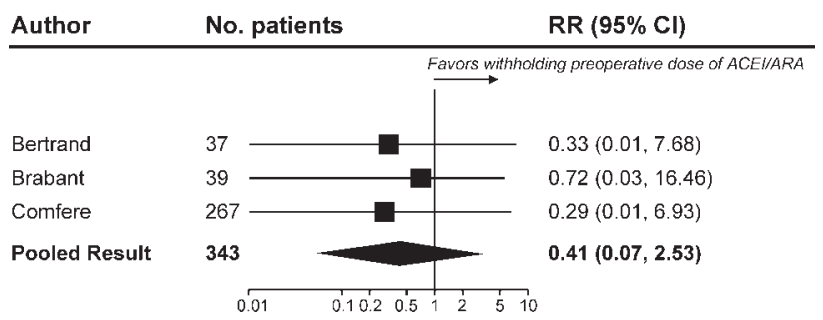

FIGURE 2. (A) Meta-analyses of included studies regarding the development of hypotension requiring vasopressors when immediate preoperative doses of ACE/ARAs are given or withheld. (One study ${ }^{10}$ did not report the number of patients in the ACEl-withheld group who required vasopressors. We used 18, the total number of patients reported to be hypotensive according to the authors' systolic pressure-based definition. Those authors ${ }^{10} d o$ report that all 12 patients in the ARA-continued group required vasopressors. Thus, our use of 12 and 18 patients in the given and withheld groups, respectively, is conservative [ie, underestimates the treatment effect].) (B) Meta-analyses of the 3 included studies that examined the effect on risk of postoperative myocardial infarction of giving versus withholding the preoperative dose of an ACEI/ARA.

The incidence of perioperative myocardial infarction was not significantly different between continuing and withheld groups (Fig. 2B); the results were consistent across trials $\left(\mathrm{I}^{2}=0 \%\right)$ but were imprecise $(\mathrm{RR}=0.41,95 \%$ CI $0.07-2.53)$. Data were insufficient for subgroup analyses.

\section{DISCUSSION}

\section{Statement of Principle Findings}

Our systematic review identified 3 randomized trials and 2 observational studies examining the clinical consequences of continuing versus deliberately withholding the immediate preoperative dose of a renin-angiotensin-aldosterone system antagonist in patients treated chronically with these agents and scheduled to undergo nonemergent surgery.
Results from pooled estimates suggest that continuing chronic therapy up until surgery may increase the risk of perioperative hypotension requiring vasopressors (Fig. 3). Otherwise, this systematic review did not identify any clinically significant consequences associated either with preoperatively withholding or continuing RAAS antagonists. We do note that all 3 of the myocardial infarctions reported occurred in patients from whom the immediate preoperative ACEI/ARA dose was withheld, although no meaningful conclusion can be inferred from so few data points.

\section{Strengths and Weaknesses of This Review}

We observed considerable variation in design quality from study to study. With the exception of hypotension, other end points were not examined uniformly in the studies comprising this review. This was due either to study design (retrospective) or to the belief that the outcomes were not likely. With 1 exception, ${ }^{11}$ patient-important end points such as myocardial infarction were noted if they occurred but not explicitly sought. Without active surveillance (serial electrocardiographic and biomarker testing), events such as myocardial infarction may remain undetected. Pain from myocardial ischemia, for example, may be masked by postoperative analgesia. Creatine kinase with muscle and brain subunits (CK-MB) may be elevated in response to extracardiac injury. Postoperative ECG findings often are nonspecific. ${ }^{12}$ Furthermore, these studies examined the immediate and short-term postoperative periods, possibly missing late-manifesting hypotension-induced or other end-organ damage. Thus, truly reliable conclusions regarding the frequency of myocardial infarction, cerebrovascular events, and other patient-important outcomes cannot be reached. Because this review includes small studies, it is particularly vulnerable to the effects of publication bias. The overall quality of the evidence we summarized makes it likely that larger rigorous trials may fail to confirm our findings. ${ }^{13-15}$ Notably, this is to our knowledge the first systematic review addressing the clinical consequences of continuing or withholding the immediate preoperative dose of ACEI/ARAs.

\section{Meaning of the Study}

Evidence exists that perioperative ACEI/ARA therapy can impair the body's already anesthesia- 
A)

\begin{tabular}{|c|c|c|}
\hline & 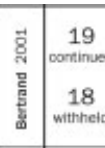 & 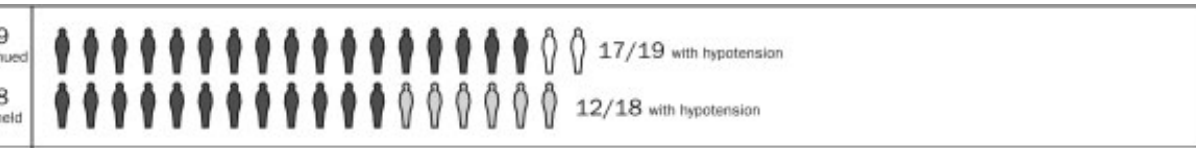 \\
\hline & \begin{tabular}{l|l}
21 \\
0 \\
0
\end{tabular} & 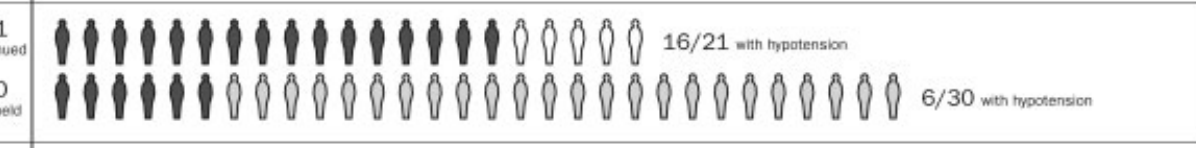 \\
\hline & 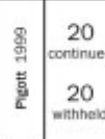 & 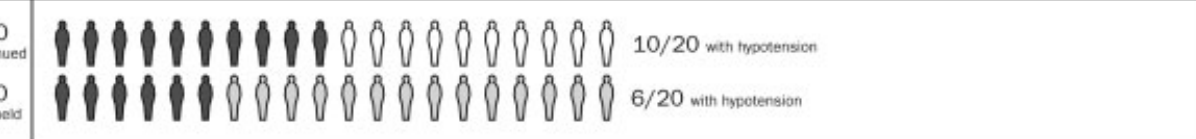 \\
\hline & & 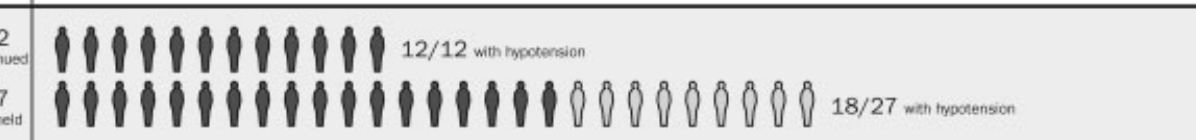 \\
\hline & 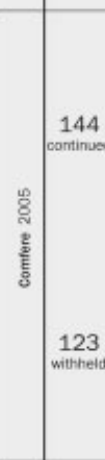 & 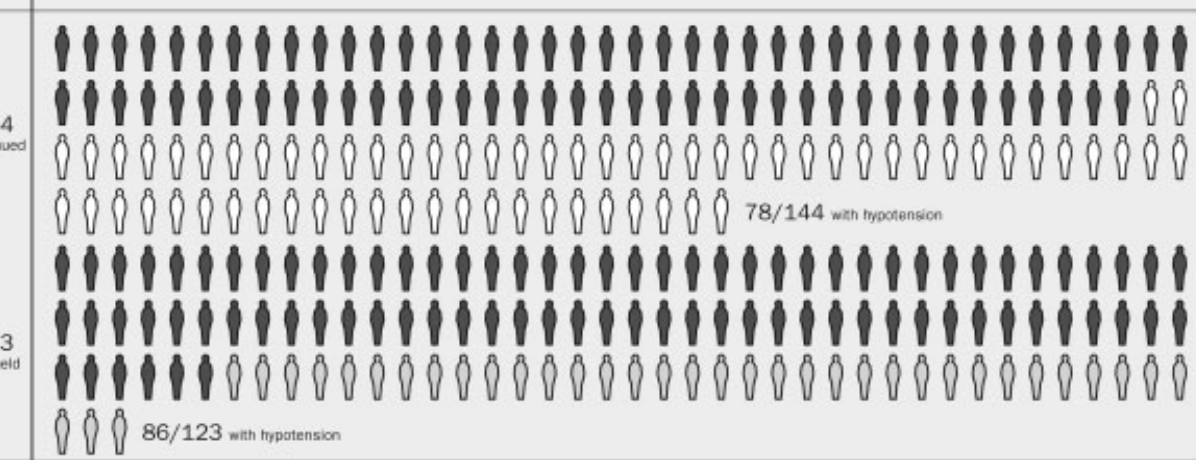 \\
\hline
\end{tabular}

B)

\begin{tabular}{|c|c|c|}
\hline ing & 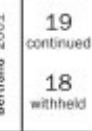 & 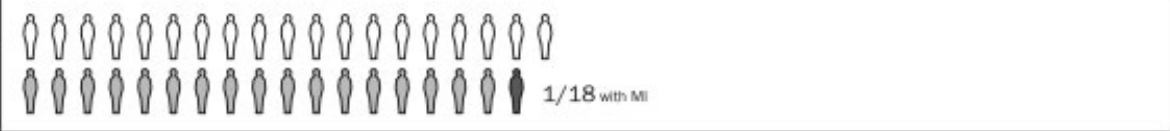 \\
\hline$g$ & 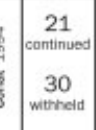 & 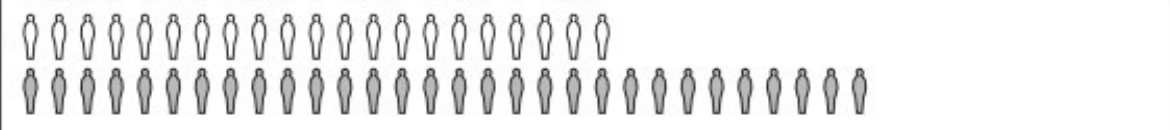 \\
\hline$\frac{9}{2}$ & $\begin{array}{l}20 \\
\text { continues } \\
20 \\
\text { miresta }\end{array}$ & 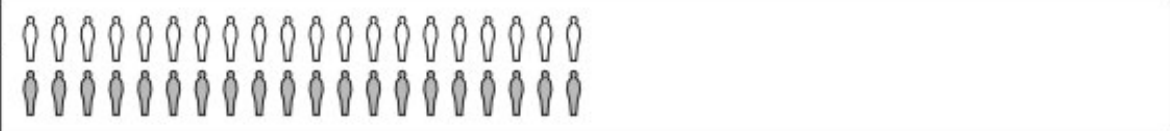 \\
\hline & & 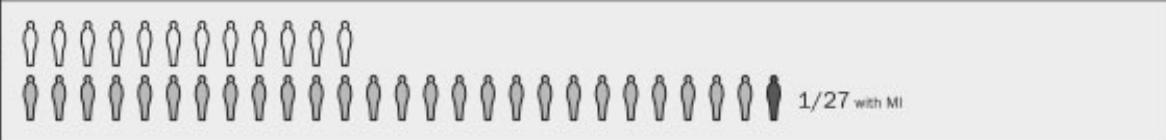 \\
\hline & 144 & 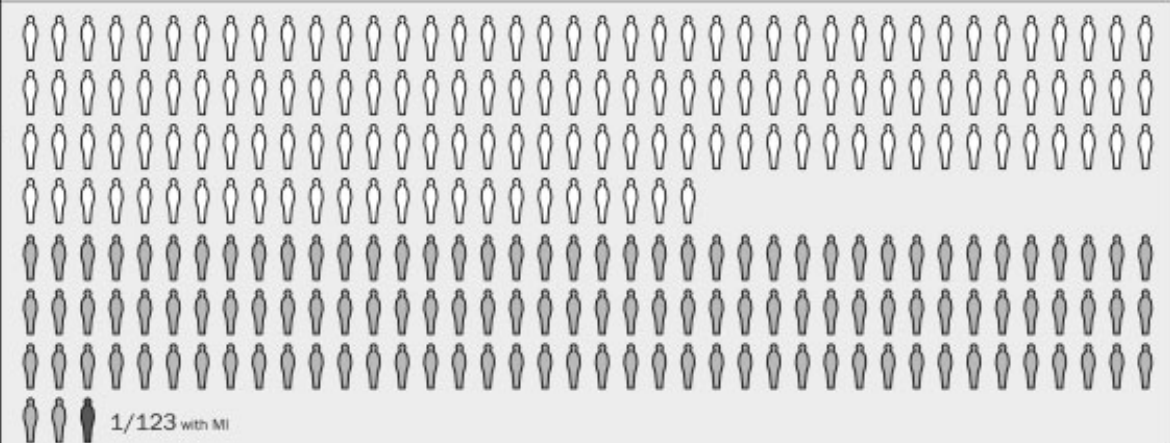 \\
\hline
\end{tabular}

FIGURE 3. Summary of shared clinical outcomes for individual studies. (A) Frequency of hypotension requiring vasopressors. (B) Frequency of myocardial infarction. 
suppressed blood pressure regulation system. Patients scheduled to undergo cardiovascular surgery may be at increased risk for the development of perioperative hypotension requiring vasopressors if the immediate preoperative ACEI/ARA dose is given. The results of this review-a review of studies that were relatively small and generally not powered to observe clinically significant consequences-do not provide sufficient evidence to support the systematic withholding or the systematic continuation of RAAS antagonists. Patients will be served best by hospitalists and other clinicians involved in perioperative care who take into account situation-specific details in making this decision. A patient at particularly high risk for the complications of a blood pressure extreme (either hyper- or hypotension) represents such an example.

For patients who receive the immediate preoperative ACEI/ARA dose and do develop perioperative hypotension, there is inadequate evidence to determine whether that hypotension leads to patient-important adverse outcomes. In fact, data from literature presently available are insufficient to reach any conclusion about long-term clinical consequences of continuing or not continuing chronic ACEI/ARA therapy. The available studies were relatively small, reported few if any events, and were not designed to measure accurately the incidence of patient-important end points.

\section{Unanswered Questions and Future Research}

Large and rigorous randomized trials could help to clarify the relationships suggested in this metaanalysis and provide valid data about the consequences of continuing versus withholding preoperative ACEI/ARA therapy. Such trials are required before strong evidence-based recommendations can be formulated.

\section{ACKNOWLEDGMENTS}

The authors are indebted to James M. Naessens, ScD, David R. Danielson, MD, and David O. Warner, $\mathrm{MD}$, for their advice during the conduct of this study. We also gratefully acknowledge Amanda Ebright, MD, for asking the original question that led to this review and Mr. Matthew Maleska for his design of summary Figure 3.
Address for correspondence and reprint requests: David J. Rosenman, MD, Mayo Clinic, 200 First Street SW, Rochester, MN 55905; Fax: (507) 2551027; E-mail: Rosenman.david@mayo.edu

Received 6 August 2007; revision received 29 November 2007; accepted 31 December 2007.

\section{REFERENCES}

1. Guyatt G, Montori V, Devereaux PJ, Schunemann H, Bhandari M. Patients at the center: in our practice, and in our use of language. ACP J Club. 2004;140:A11-A12.

2. Moher D, Cook DJ, Eastwood S, Olkin I, Rennie D, Stroup DF. Improving the quality of reports of meta-analyses of randomised controlled trials: the QUOROM statement. Lancet. 1999;354:1896-1900.

3. Wells GA, Shea B, O'Connell D, et al. The NewcastleOttawa Scale (NOS) for assessing the quality of nonrandomized studies in meta-analysis. Ottawa Health Research Institute, University of Ottawa, Ontario, Canada. http:// www.ohri.ca/programs/clinical_epidemiology/oxford.htm. Accessed: March 21, 2006.

4. DerSimonian R, Laird N. Meta-analysis in clinical trials. Control Clin Trials. Sep 1986;7:177-188.

5. Michael J. Sweeting AJS, Paul C. Lambert. What to add to nothing? Use and avoidance of continuity corrections in meta-analysis of sparse data. Stat Med. 2004;23:1351-1375.

6. Higgins JPT, Thompson SG. Quantifying heterogeneity in a meta-analysis. Stat Med. 2002;21:1539-1558.

7. Altman DG, Bland JM. Statistics notes: Interaction revisited: the difference between two estimates BMJ. 2003;326:219

8. Coriat P, Richer C, Douraki T, et al. Influence of chronic angiotensin-converting enzyme inhibition on anesthetic induction. Anesthesiology. 1994;81:299-307.

9. Comfere T, Sprung J, Kumar MM, et al. Angiotensin system inhibitors in a general surgical population. Anesth Analg. 2005;100:636-644.

10. Brabant SM, Bertrand M, Eyraud D, Darmon PL, Coriat P. The hemodynamic effects of anesthetic induction in vascular surgical patients chronically treated with angiotensin II receptor antagonists. Anesth Analg. 1999;89:1388-1392.

11. Bertrand M, Godet G, Meersschaert K, Brun L, Salcedo E, Coriat P. Should the angiotensin II antagonists be discontinued before surgery? [see comment]. Anesth Analg. 2001;92:26-30.

12. Zipes. Braunwald's Heart Disease: A Textbook of Cardiovascular Medicine. 7th ed. New York: Saunders, an imprint of Elsevier; 2005.

13. Montori V, Guyatt G. Summarizing the Evidence: Publication Bias. Chicago: AMA Press; 2002.

14. Cappelleri JC, Ioannidis JP, Schmid $\mathrm{CH}$, et al. Large trials vs meta-analysis of smaller trials: how do their results compare? [see comment]. JAMA. 1996;276:1332-1338.

15. Egger M, Smith GD, Schneider M, Minder C. Bias in meta-analysis detected by a simple, graphical test. BMJ. 1997; 315:629-634.

16. Fleisher LA. Preoperative evaluation of the patient with hypertension. JAMA. 2002;287:2043-2046.

17. Pigott DW, Nagle C, Allman K, Westaby S, Evans RD. Effect of omitting regular ACE inhibitor medication before cardiac surgery on haemodynamic variables and vasoactive drug requirements. Br J Anaesth. 1999;83:715-720. 